Functional

Ecology 1993

7, 513-521

\title{
Optimal adult growth of Daphnia in a seasonal environment
}

\author{
B. E. TAYLOR* and W. GABRIEL \\ Max-Planck-Institut für Limnologie, Abteilung Ökophysiologie, Postfach 165, W-2320 Plön, Germany
}

\begin{abstract}
Summary
1. The cladoceran Daphnia serves as an example of an iteroparous organism, with overlapping generations, that is capable of substantial adult growth. The life history of Daphnia was modelled as the consequence of a series of decisions about allocation of energetic resources to growth and reproduction.
\end{abstract}

2. We used numerical methods to find resource allocation patterns that maximized fitness of Daphnia in a temporally variable environment. Temporal variation was modelled as alternating active and dormant seasons; length of the active season was uniformly distributed. Fitness was measured by the geometric mean of resting eggs produced at the end of the active season. We examined effects of mean and range of the active season on the optimal life history; we also examined effects of increasing (invertebrate predation), constant (non-selective) and decreasing (fish) size-specific survival rates. For comparison, we found resource allocation patterns that maximized fitness in a constant environment, where fitness was measured by the intrinsic rate of increase $r$.

3. Life histories optimized for seasonal environments generally showed earlier maturity and greater adult growth than those optimized for constant environments. Adult growth occurred with non-selective predation, and even with fish predation, conditions under which it does not occur in the optimal life histories for constant environments.

4. Greatest size at maturity and adult growth occurred in life histories optimized to invertebrate predation in seasonal environments. Smallest size at maturity and least adult growth occurred in life histories optimized to fish predation.

5. In the optimal life histories, size at maturity generally increased with mean length of the active season. Adult growth reached a maximum for mean seasons of length equal to about one-half to one life span of Daphnia.

6. Increasing the variation in season length decreased adult growth in the optimal life history, but had little effect on size at maturity.

7. We expect that life histories are adapted to the long-term average of season length and its variation. If the animals can detect the type of predator, selection could favour phenotypic variation in resource allocation.

Key-words: Life history, predation, resource allocation, variable season

Functional Ecology (1993) 7, 513-521

\section{Introduction}

The life histories of plants and animals display an enormous variety of pattern in growth and reproduction, challenging the observer to explain or predict how the patterns relate to success under various ecological circumstances. This is the general life-his-

*Present address and to whom reprint requests should be addressed: Dr B. E. Taylor, Savannah River Ecology Laboratory, Drawer E, Aiken, South Carolina 29802, USA. tory problem addressed by Cole (1954), León (1976), Stearns (1976), Schaffer (1983), and many others. Presuming that these patterns are shaped by natural selection, the theoretician builds models to translate observable life-history traits into schedules of survival and reproductive rates and thus to assess their potential adaptive significance.

In this paper we consider a trait, growth after maturity, that is a prominent feature of the life history of the crustacean Daphnia. This trait is common to many invertebrates, poikilotherm verte- 
brates, and plants. For organisms whose productive capacity and other vital rates depend strongly on body size, a resource allocation model provides a plausible mechanism for specifying trade-offs between growth and reproduction and evaluating their demographic consequences, from which potential adaptive significance is inferred.

Resource allocation models have been applied to study growth after maturity for both plants and animals. The models differ in many features, including costs and benefits associated with growth, demands on the allocatable resource, generational overlap, and pattern of environmental conditions.

When production of offspring can occur continuously, the problem of allocating resources between growth and reproduction becomes a standard problem in optimal control. With on-off or bang-bang control, the organism stops growing and begins reproducing when it reaches some critical size. The alternative is a transitional period during which the organism grows and reproduces simultaneously, before devoting the resource entirely to reproduction. For deterministic models of life histories that feature overlapping generations, the intrinsic rate of increase $r$ is always maximized by an on-off control if reproduction and growth depend linearly on resource allocation and mortality is constant (León 1976) or size-dependent (Perrin 1992; the model allows allocation to more than one kind of somatic structure). If reproduction is a non-linear function of resource allocation, the optimal strategy may include a period of intermediate allocation (León 1976; Sibly, Calow \& Nichols 1985). Intermediate allocation may also occur if mortality is a function of growth, but not if it is a function only of body size (Sibly et al. 1985; the latter result is a simple extension of their analysis).

Different models for the demographic consequences of the life history are needed if the production of offspring can occur continuously, but only within a season of limited length. When generations do not overlap (annual plant, for example), net reproductive output $R_{\mathrm{o}}$ is appropriately maximized as the measure of fitness (see Schaffer 1983); when generations do overlap but recruitment occurs only at the beginning of the growing season (perennial plant), the intrinsic rate of increase $r$ is again the measure of fitness, but is calculated using the discrete form of the renewal equation. When the responses to allocation are linear and mortality is constant, the optimal allocation for the annual plant is an on-off pattern (Vincent \& Pulliam 1980; Schaffer 1983; Iwasa \& Roughgarden 1984). This pattern also holds for perennial plant models when the intrinsic rate of increase is maximized and mortality is constant (Schaffer 1983), but if the critical size is reached mid-season, reproduction may be lower in the first year than in subsequent years (Pugliese 1988a). Conditions that yield intermediate allocation include random variation in season length for an annual plant
(King \& Roughgarden 1982) and non-linear return on the investment in reproduction for an annual plant (Kozlowski \& Ziólko 1988) and a perennial plant (Pugliese 1988b). For a perennial plant, reproduction is effectively a discrete process, because it is not completed until the seeds germinate at the beginning of the next growing season. Pugliese (1988a) showed that solutions for the discrete model of a perennial plant thus apply also to the corresponding continuous model. For the discrete time model, Pugliese (1987) showed that reproduction may begin before the critical size is reached if production is an increasing, but not concave, function of size or if survival rate increases as a function of size.

From this diverse collection of optimal resource allocation strategies, we can extract a few general predictions about conditions that may favour adult growth. These conditions include non-linearities in response of reproduction or growth to resource allocation and, if birth (or germination or hatching) occurs at discrete intervals, increasing size dependency of survival rate. Unpredictable temporal variation of the environment can also produce adult growth, but its effects have been explored only for season length for an annual plant.

Studies of resource allocation models for Daphnia yield one set of results that fit, by analogy with previously studied models, into this broader theoretical context and two sets that lie outside its domain. In the Taylor-Gabriel models (Gabriel \& Taylor 1991; Taylor \& Gabriel 1992), reproduction occurs at discrete intervals. When the intrinsic rate of increase is maximized, growth after maturity occurs if survival rate increases with body size, but not if it decreases or remains constant with body size (Taylor \& Gabriel 1992). Body size of Daphnia corresponds to storage reserve in the perennial plant of Pugliese (1987), other general conditions match, and the results fit his conclusions about conditions under which growth after maturity may occur. With the same model, Gabriel \& Taylor (1991) found that alternating schedules of increasing and decreasing size-specific survival rates favoured life histories with substantial adult growth. The intrinsic rates of increase with each survival rate schedule were averaged to approximate fitness. The measure of fitness neglects the effects of transitions in age structure when the survival rate schedules change; it becomes more appropriate as the interval under each schedule becomes longer. This pattern of environmental variation has not been studied for other organisms. With a model that treats reproduction as a continuous function, Gabriel (1982) found that adult growth maximized net lifetime reproduction $R_{\mathrm{o}}$ when survival rate decreased with body size, but not when mortality was constant. The results for constant mortality are predicted by optimal control models for the annual plants, but decreasing size-dependent survival rate has not been considered. 
Daphnids typically produce multiple, overlapping generations during a growing season which may last from weeks to months. To study effects of environmental variation at time scales ecologically important to Daphnia, we model an environment in which a growing or active season alternates with a season during which Daphnia remains dormant as resting eggs. This model represents a class of environmental variation - extreme shifts in environmental conditions within the lifetime of the animal - whose great importance ecologically and evolutionarily is indicated by the prevalence among cladoceran populations of the capacity for producing resting eggs. We examine effects of both mean and variation of season length on the optimal life history under several forms of size-dependent mortality. With these results, we discuss how the life history might be shaped to accommodate environmental variation and how adaptations to certain patterns of variation might enhance or degrade responses to other conditions. We also consider the generality of the predictions from models that assume constant environmental conditions.

\section{The organism}

Cladocerans of the genus Daphnia are small $(0 \cdot 3-$ $5.0 \mathrm{~mm}$ ) planktonic crustaceans that are often abundant in lakes and ponds (Hebert 1978; Lynch 1980; Threlkeld 1987). Reproduction is iteroparous $(\geqslant 10$ broods), and adult growth can be substantial $(1 \cdot 7 \times$ in length, $4 \times$ in mass). Populations can increase rapidly $\left(r \geqslant 0 \cdot 3 \mathrm{day}^{-1}\right)$ when food (mainly algae) is plentiful and predators (fish, amphibians, aquatic insects and larger crustacean zooplankton) are scarce. For many Daphnia populations, resting eggs provide a refuge from adversities, such as insufficient food, extreme temperatures, intensified predation, or even drying of the habitat. Resting eggs, typically two per brood, are produced in response to cues, such as photoperiod, crowding, or diminishing food, that signal the onset of poor conditions (Hebert 1978; Kleiven, Larsson \& Hobæk 1992). The favourable season may last for several months in temperate lakes, but can be much briefer in habitats such as arctic tundra ponds or temporary ponds.

\section{Models}

Daphnia has often been modelled and the models usually incorporate adult growth. Some excellent, detailed recent models describe resource allocation as a function of size, nutritional state and competing physiological processes (for example, Gurney et al. 1990; Hallam et al. 1990). A goal of these models is to produce a model organism whose demographic characteristics, under varying environmental conditions, closely match those of the real Daphnia. Although our model is based on laboratory data for Daphnia (data which were also used to develop the models cited), our purpose here is different. We wish to understand better the selective pressures which, we presume, have shaped evolution of the physiological mechanisms which direct the growth of real Daphnia. To that end, we manipulate the allocation process to explore its effect on fitness.

\section{DAPHNIA}

The Daphnia model is based on experimental lifetable data for D. pulex (Taylor 1985), and factors converting energy to mass were derived from measurements in Richman (1958). The experimental Daphnia were raised at $20^{\circ} \mathrm{C}$ with algal food supplied at $1 \mathrm{mg}$ carbon $\mathrm{l}^{-1}$, a concentration well above levels that would limit growth and reproduction. The model described here is very similar to that of Taylor \& Gabriel (1992).

The model life history for Daphnia consists of two juvenile instars $\left(J_{1}, J_{2}\right)$ and eleven adult instars $\left(A_{0}\right.$, $\left.A_{1}, A_{2}, \ldots, A_{10}\right)$. Instar $A_{0}$ is the pre-reproductive instar. Instar duration is $D_{J}=1.0$ days for juveniles and $\mathrm{D}_{A}=2.5$ days for pre-reproductives and adults, giving a life span of 29.5 days. Daphnia normally complete three to four juvenile instars and one pre-reproductive instar of 1-2 days each; for computational convenience we reduced the number, but not the time span. Body length $L(\mathrm{~mm})$ during instars $J_{1}$, $J_{2}$ and $A_{0}$ is fixed $\left(L_{J_{1}}=0.72 \mathrm{~mm}, L_{J_{2}}=1.07 \mathrm{~mm}\right.$, $L_{A_{0}}=1.42 \mathrm{~mm}$ ); length during subsequent instars is determined by the allocation to growth.

Net production $E$ (J per adult instar) is a function of body mass $W$ ( $\mu \mathrm{g}$ dry mass):

$E=2 \cdot 73\left(1-\frac{1}{0 \cdot 731+0 \cdot 0318 W}\right)$ eqn 1

In each instar $A_{k}(k=0, \ldots, 9)$, a proportion $\alpha_{k+1}$ of this energy can be allocated to reproduction. This proportion determines the number of eggs $m_{k+1}$ laid at the beginning of the next instar:

$m_{k+1}=\frac{\alpha_{k+1} C_{\text {rep }} E_{k}}{W_{\text {egg }}}$ eqn 2

where $C_{\text {rep }}=40 \mu \mathrm{g} \mathrm{J}^{-1}$ converts net production to reproductive mass and $W_{\text {egg }}=2 \cdot 5 \mu \mathrm{g}$ is the dry mass of an egg. The remainder is allocated to growth, which determines body mass $W$ and length $L$ at the beginning of the next instar:

$W_{k+1}=W_{k}+\left(1-\alpha_{k+1}\right) C_{\text {som }} E_{k}$ eqn 3

where $C_{\text {som }}=60 \mu \mathrm{g} \mathrm{J}^{-1}$ converts net production to somatic mass, and:

$L=\frac{\log (W-1)}{1 \cdot 2}$

eqn 4

The trade-off between growth and reproduction is illustrated in Fig. 1. The eggs $m_{k+1}$ are carried in the mother's dorsal brood chamber, where they hatch 
516

B. E. Taylor \&

W. Gabriel

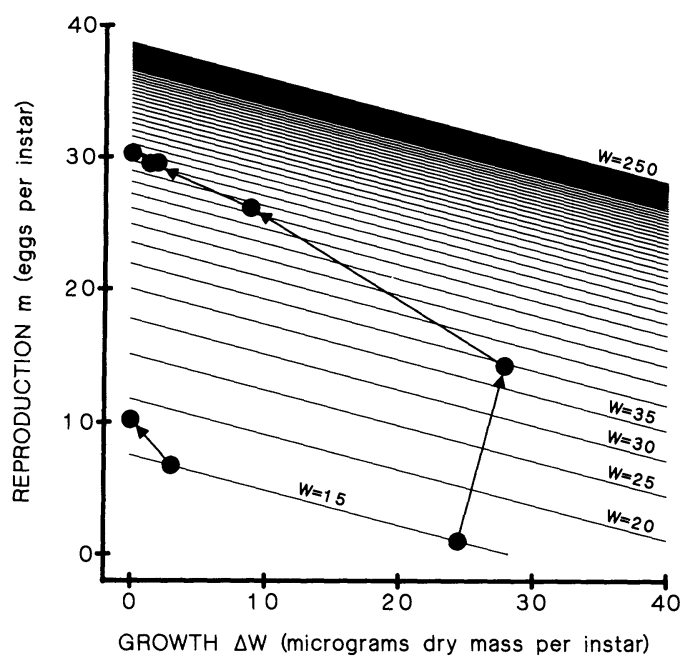

Fig. 1. The trade-off between growth and reproduction. Lines show trade-off functions for animals of dry masses $W$ from 15 to $250 \mu \mathrm{g}$ at $5-\mu \mathrm{g}$ intervals. Filled circles show the allocation in 10 successive instars for two optimized life histories. In the optimal life history for fish predation in a season of $30 \pm 5$ days, one instar of intermediate allocation is followed by nine instars of allocation entirely to reproduction (point at $\Delta W=0$ ). In the optimal life history for invertebrate predation in a season of $30 \pm 5$ days, five intars of intermediate allocation are followed by five instars of allocation entirely to reproduction.

and progress through embryonic development. Freeswimming juveniles are released from the brood chamber at the end of the adult instar $k+1$, when the mother's age is:

$x_{k+1}=2 D_{J}+(k+2) D_{A}$

eqn 5

Survival rate $p$ over an instar of duration $D$ depends on the length of the animal:

$p=(a L+b)^{D / 2 \cdot 5}$

eqn 6

Predation by invertebrates, such as larvae of the phantom midge Chaoborus, causes the survival rate of Daphnia to increase with size $(a=0.117$, $b=0.316$ ); predation by fish causes the survival rate to decrease $(a=-0 \cdot 117, b=0 \cdot 984)$. A non-selective predator or other agent of mortality causes a constant survival rate $(a=0, b=0 \cdot 650)$. (Please note that not all invertebrates or all fish have similar general effects on survival rates of Daphnia.) Survivorship $l_{k}$ to the end of adult instar $k$ is:

$l_{k}=p_{J_{1}} p_{J_{2}} \prod_{i=1}^{h} p_{i}$

eqn 7

\section{THE SEASONAL ENVIRONMENT}

Temporal variation of the model environment consists of a sequence of alternating seasons of activity and dormancy for Daphnia. For convenience, we may imagine that the active season occurs annually. At the beginning of the active season, daphnids hatch from resting eggs. They and their offspring grow and reproduce until receiving a cue signalling the season's end, when each reproducing animal forms two resting eggs. For an adult of instar $k$ to be counted as reproducing, the investment $\alpha_{k}$ must yield at least one egg $\left(m_{k} \geqslant 1\right)$ under conditions of normal reproduction. Resting eggs are produced at the beginning of the reproductive instar. All resting eggs survive with probability $s$ to hatch at the beginning of the next season of activity; $s$ is independent of the length of the dormant season.

The length of the active season has a uniform distribution, described by a mean and range. When the range is zero, the length of the active season is fixed, and the temporal variation of the environment is completely predictable: each new active season is the same as the last. When the range is greater than zero, the temporal variation is not completely predictable.

We studied effects of both mean and range of the active season on the optimal life history. The active season was measured in 0.5 day units, corresponding to the time step of population growth simulations for Daphnia. The mean and range of the distribution were chosen so that the minimum active season was $\geqslant 4.5$ days, the earliest age at which the model Daphnia can produce resting eggs. The mean was extended up to 50 days (20 adult instars or $1 \cdot 7$ lifetimes).

Setting the length of the active season also sets the length of its annual complement, the dormant season. The length of the dormant season, however, has no direct effect on success of the resting eggs, because $s$ is independent of length of the dormant season.

\section{FITNESS}

For a single active season, the success of a life history is measured by the number of resting eggs $N$ produced at the end of the active season by an animal and her descendants. Over a series of $n$ years, success is measured by the number of resting eggs $H_{n}$ produced at the end of the last active season and surviving to hatch at the beginning of the next active season:

$H_{n}=H_{0} \prod_{i=1}^{n} N_{i} s_{i}$

eqn 8

where the hatch of resting eggs at the beginning of the first year is $H_{0}=1$. If we assume that the $s_{i}=s$ are independent of season or life history, fitness $F$ is given by the geometric mean of the $N_{i}$ :

$\log (F)=s \lim _{n \rightarrow \infty} \frac{1}{n} \sum_{i=1}^{n} \log \left(N_{i}\right)$

eqn 9 
Optimal growth of

Daphnia

Because $s$ is simply a scaling factor, we can set $s=1$ without loss of generality. If the frequency distribution of values of $N$ is known:

$\log (F)=\sum_{j=1}^{m} f_{j} \log \left(N_{J}\right)$

where $N$ has $m$ possible values and each value $N_{j}$ occurs with frequency $f_{j}$.

For our model Daphnia, $N$ is a function of the length of the active season, the growth and fecundity schedule determined by the resource allocation pattern, and the size-dependent survival rate set by the type of predator. We calculated $N$ using a population growth simulation with time step $\Delta t=0.5$ day for each active season length in the set described by the distribution of active seasons. We then calculated fitness according to equation 10 . For a particular kind of predation and distribution of active seasons, the optimal life history is the one that maximizes this measure of fitness

The measure of fitness for a stable environment is the intrinsic rate of increase $r$ defined by the renewal equation (Keyfitz 1977):

$1=\sum_{k=1}^{10} l_{k} m_{k} \mathrm{e}^{-r x_{k}}$

eqn 11

We used standard numerical optimization methods to find resource allocation schedules that maximized fitness for the model Daphnia. Values of $\alpha$ were constrained to the range 0 to 1 . Testing the solution with variations of the resource allocations $\alpha$ insured that each result represented a local optimum. To determine that the local optima represented global optima, within the specified constraints, behaviour of the optimal solutions was further tested by altering initial values of the optimized variables, by changing the set of optimized parameters and by varying parameters of the model.

\section{Results}

In the model for Daphnia, variations in resource allocation can produce variations in size and fecundity in any of the 10 adult instars. Optimizing the life history for a season of a particular length entails maximizing the contributions to resting egg production from the initial cohort and any generations of offspring that mature during the season. In the optimal life histories, except in very short seasons, most of the resting eggs were produced by animals of instar $A_{1}$, the first reproductive instar, but not necessarily of the youngest generation. We summarized the optimal life histories by the adult size range, from size at maturity (the instar of first reproduction) to maximum size. Size at maturity depends, of course, on allocation $\alpha_{1}$ in the pre-reproductive instar $A_{0}$ (and one or more of the subsequent allocations, if first reproduction is postponed by one or more instars), while maximum size depends on the whole history of allocation.

In an environment with alternating seasons of activity and dormancy, the predator and the mean and range of the distribution of the active season each had large effects on the optimal life history (Fig. 2). Size at maturity and subsequent growth were greatest with invertebrate predation and least with fish predation. These differences held for nearly all of the season distributions tested, the exceptions occurring with short seasons of fixed length.

When season length was fixed, small changes in length of the active season produced dramatic changes in the optimal life history (Fig. 2b). Very short fixed seasons favoured complete allocation to reproduction, whatever the form of predation. Growth before and after first reproduction increased with season length, producing greater size at maturity and greater adult growth. Adult growth was greatest in seasons of moderate length, up to $\sim 5$ weeks. With longer seasons, adult growth decreased; it also occurred less frequently in the later adult instars.

Variability in season length smoothed the response of the optimal life history to changes in the mean length of the active season (Fig. 2a). Pre-reproductive growth, indicated by size at maturity, increased slightly with mean season; adult growth was greatest when the mean season was fairly short ( $<3-4$ weeks). The variability in season length had little overall effect on size at maturity (for fixed seasons of 20-50 days, mean size at maturity was $1.95 \mathrm{~mm}$; for mean seasons of $20 \pm 5$ days to $50 \pm 5$ days, mean size at maturity was $1.97 \mathrm{~mm}$ ). The variability had a much stronger effect on adult growth, diminishing it and shifting it towards earlier instars. Under fish predation, adult growth was lost entirely when the range increased to \pm 7.5 days (not shown) or \pm 10 days, although adult growth was retained in some life histories optimized to mean seasons with \pm 5 day ranges (not shown).

In contrast to the optimal life histories for the constant environment (Fig. 2a), adult growth occurred under all predation models in life histories optimized for the seasonal environments. As season mean or range increased, the optimal life history for the seasonal environment became more similar to the corresponding optimal life history for the constant environment, although reproduction was usually initiated at a smaller size in the seasonal environment. With non-selective and fish predation, the similarities were close. With invertebrate predation, pre-reproductive growth was extended through instar $A_{1}$ in the optimal life history for the constant environment. For the seasonal environment, the animal made a small investment in eggs for instar $A_{1}$ to meet the criterion for initiation of reproduction. Thus, the earliest possible instar produced resting eggs at the end of the season. (When resting egg 

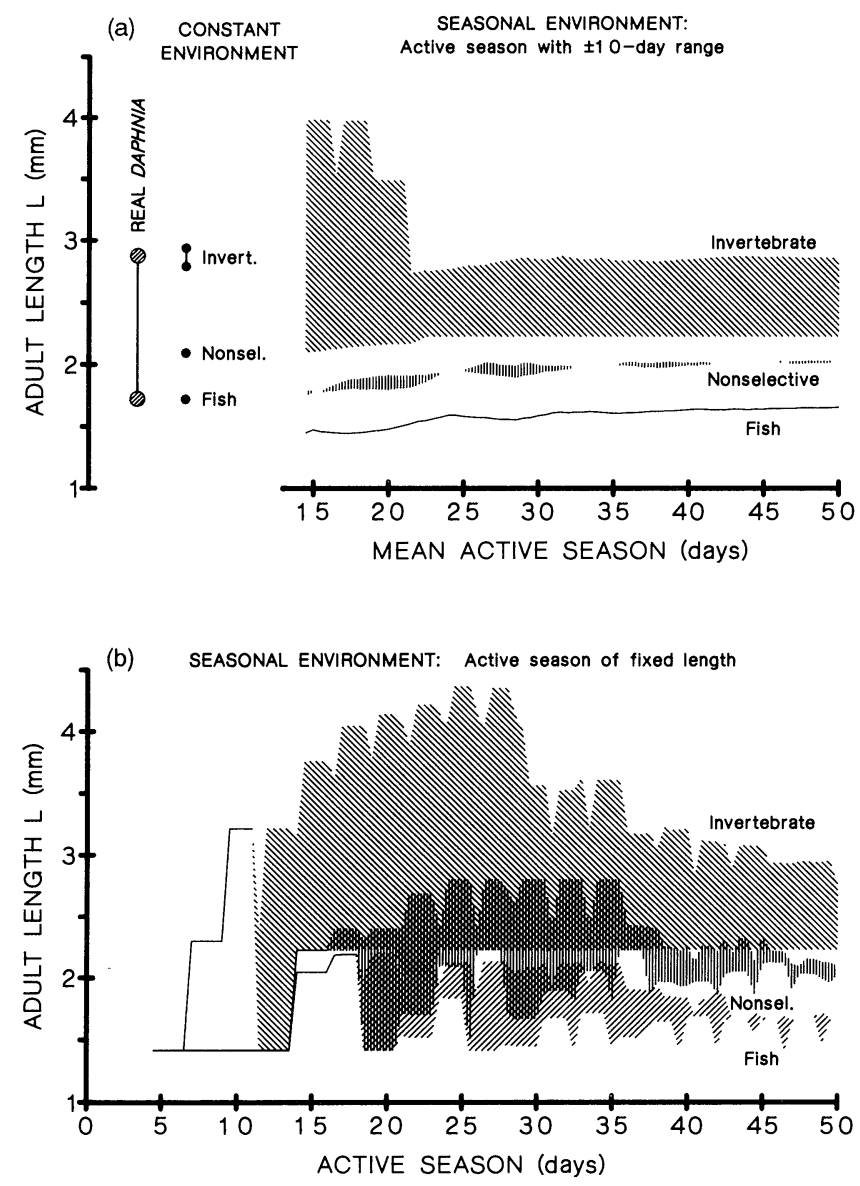

Fig. 2. Optimal size of adult Daphnia. Length from size at maturity to maximum size is shown for life histories optimized to invertebrate, non-selective and fish predation. (a) Length of the active season is distributed uniformly with a range of \pm 5 days around the mean. (b) Length of the active season is fixed ( \pm 0 day range). For comparison, optimal adult size of Daphnia for each form of predation in a constant environment is shown in (a). Adult size of the real D. pulex (Taylor 1985) that provided the basis for the model is shown on the far left in (a).

production was allowed unconditionally in $A_{1}$, reproduction was usually delayed by one instar in the seasonal environment, but size at maturity remained generally smaller, and adult growth greater, than in the constant environment.)

The various optimal life histories would appear different to an ecologist collecting measurements of animals from field populations. Are these differences in growth patterns important demographically? We addressed this question by measuring performances of optimized life histories in single seasons of non-selective predation; this may be interpreted as sensitivity to an unexpected season.

The mean season to which the life history was optimized did not have a large effect on performance in an unexpected season (Fig. 3a), unless the life history was optimized to a short season ( 15.5 days in our example). For all of the tested life histories, except the one optimized to the shortest mean season, performance was generally better in longer test seasons. The tests of life histories optimized to a mean season with various ranges showed a similar pattern (Fig. 3b). The life history optimized to a fixed season performed poorly in most of the test seasons (medians $<85 \%$ of maximum resting eggs in the three groups of test seasons). Performances of life histories optimized to season ranges greater than \pm 0 days were similar within each group of test seasons, and performances were better in longer seasons.

A mismatch between the predator and the life history yielded poor performance in test seasons of any range of lengths (Fig. 4). The life history optimized to invertebrate predation (medians $<70 \%$ of maximum resting eggs in the three groups of test seasons) fared worse under non-selective predation than did the life history optimized to fish predation (medians $<80 \%$ ).

\section{Discussion}

Daphnia life histories optimized for environments with alternating seasons of activity and dormancy differ generally in two important features from those optimized for constant environments. In seasonal environments, the model daphnids mature earlier, an effect that is strongest in the life histories optimized 

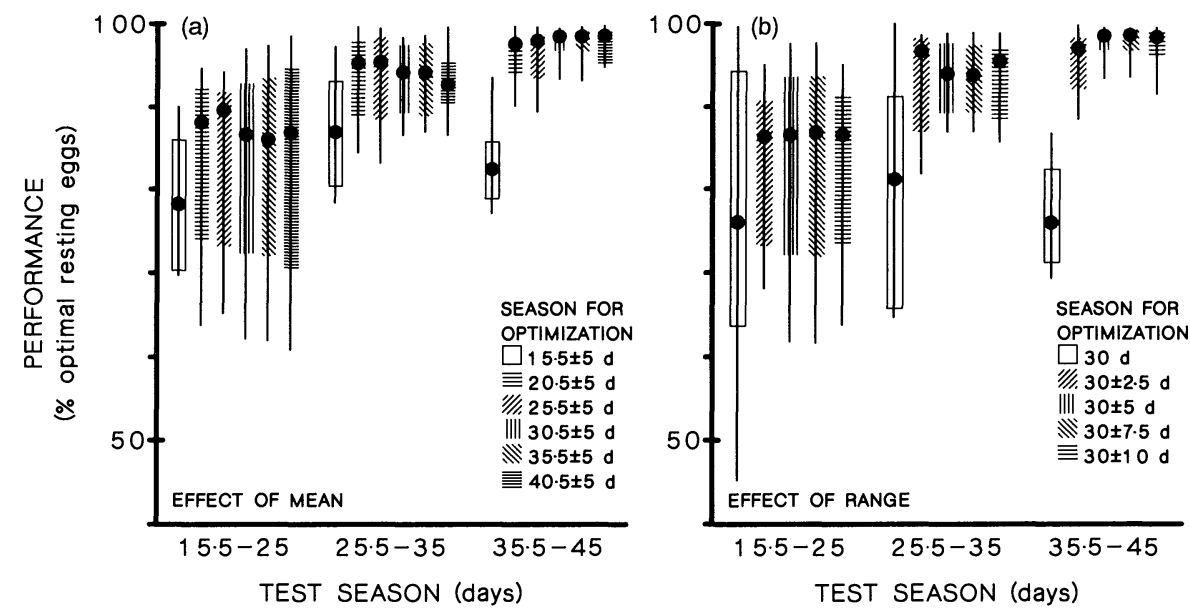

Fig. 3. Effect of mean and range for the optimized life histories on performance in a single season. Life histories were optimized to and tested with non-selective predation. Performance is measured as a percentage of the maximum possible number of resting eggs, produced by the optimal life history for that season. Median, quartiles and range of performance are plotted for each group of 20 test seasons. Within each group, test seasons were chosen at $0 \cdot 5$-day intervals corresponding to the time steps of the model.

to invertebrate predation. In seasonal environments, the model daphnids grow more extensively as adults. This effect is also most pronounced with invertebrate predation, where larger size improves survival rate as well as productive capacity. Adult growth occurs with non-selective predation, and even with fish predation, conditions under which it does not occur in the optimal life histories for constant environments (Taylor \& Gabriel 1992; results here).

Our new results contribute to the list of conditions (Perrin, Ruedi \& Saiah 1987) that may explain why adult Daphnia, and other organisms, grow. However, the optimal life history for Daphnia in the seasonal environment demonstrates a response to environmental variation that appears contrary to an earlier result on environmental variability. In King \& Roughgarden's (1982) model for an annual plant, adding variation in season length caused intermediate allocation and, thus, adult growth, and increasing variability in season lengthened this period of intermediate allocation. For Daphnia, the greatest adult growth occurs when the life history is optimized to a season of fixed length; increasing variability in season length decreased adult growth. (Note that each new cohort of Daphnia within a season experiences a different time to end-of-season and that the performance of this collection of cohorts is maximized.) Obviously, environmental variation can have different results with different models and measures of fitness; the basis for generalization about environmental variation is still quite limited.

For natural populations, the length of the favourable season is usually uncertain. For example, $D$. laevis in a temporary pond enjoyed seasons from hatching to resting egg production of $\approx 8$ and $\approx 4$ weeks in successive years (Taylor \& Mahoney 1990).
Even if physical events are closely replicated between years, variation among individuals in characteristics such as time of hatching may increase variability in the season experienced by different members of the clone. According to our model, optimizing to a fixed season is risky, because the probability of poor performance in a season of a different length is high. However, performance in an unexpected season is not very sensitive to either the mean or the range of the optimized season, so long as the mean season is

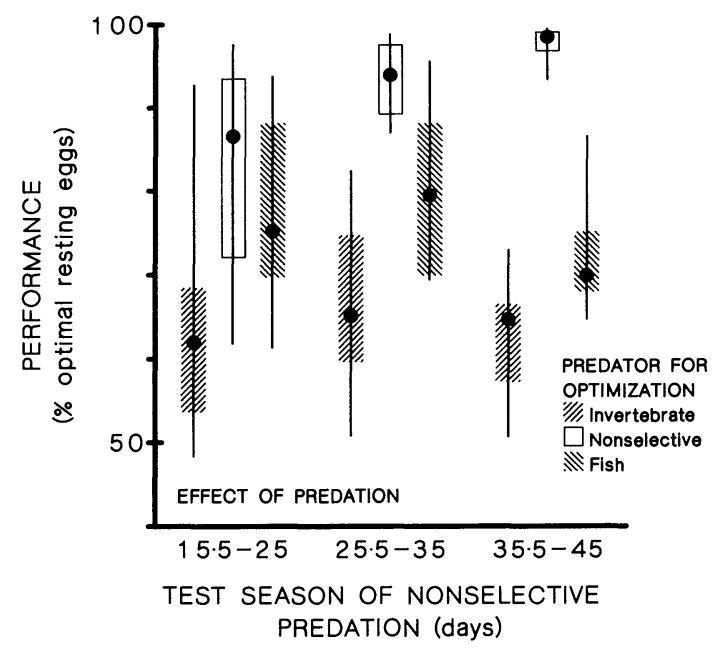

Fig. 4. Effect of predation for the optimized life histories on performance in a single season of non-selective predation. Life histories were optimized to an active season of $30 \pm 5$ days. Performance is measured as a percentage of the maximum possible number of resting eggs, produced by the optimal life history for that season. Median, quartiles and range of performance are plotted for each group of 20 test seasons. Within each group, test seasons were chosen at $0 \cdot 5$-day intervals corresponding to the time steps of the model. 
not short and the variability is not zero. For natural populations, the cost in fitness of imprecise adjustment of the life history to the long-term distribution of seasons is probably not great.

Our model represents the response of single genotype expressed as a single phenotype. Natural populations of Daphnia can exhibit great phenotypic plasticity (reviewed by Jacobs 1987), which is expressed in resource allocation and life-history traits as well as morphology. Recent work (for example, Spitze 1992; Stibor 1992) shows that chemical signals of the presence of specific types of predators may cause Daphnia life histories to change in directions consistent with the predictions of our models. Our results suggest that the performance of a life history is sensitive to the predation model, and that the cost in fitness of adaptation to the wrong form of selective predation can be large. Obviously, phenotypic plasticity could confer enormous advantages in fitness. By analogy to the reaction norms for environmental tolerance (Gabriel \& Lynch 1992), the evolution of directed plastic responses would be favoured especially when the variation of predation pressure between generations is large compared to the variation within generations. This is probably often the case: successive generations of Daphnia during a season can experience extreme shifts in size-specific predation risks if, for example, fish replace invertebrates as the dominant predators. We predict that the degree of phenotypic plasticity of Daphnia will correlate with temporal variability in predation risk.

Daphnia can use environmental cues to predict the impending end of a favourable season, but they probably cannot predict its length at the outset. We therefore expect that genetically determined values for the resource allocation patterns are adapted somehow to the long-term average of season length and its variation. We expect that plasticity is adapted to the long-term variation in predation schedules, but its effectiveness would have limits. Extreme variation in predation pressure probably causes clonal succession, with year-to-year persistence of the clones depending on resting stages. These problems need further experimental investigations, to characterize forms, costs and limits of plastic responses, as well as theoretical study.

\section{Acknowledgements}

B.E.T. is grateful for fellowships from the Max Planck Society. This research was also supported in part by contract DE-AC-09-76SR00819 between the United States Department of Energy and the University of Georgia and by grant DE-FG0989ER60881 from the DOE-OHER Theoretical Ecology Program. We thank C. W. Burns, P. Calow, W. Lampert, D. L. Schultz, H. Stibor, L. J. Weider, the
SREL life-history discussion group and an anonymous reviewer for helpful comments.

\section{References}

Cole, L.C. (1954) The population consequences of life history phenomena. Quarterly Review of Biology 29, 103-137.

Gabriel, W. (1982) Modelling reproductive strategies of Daphnia. Archiv für Hydrobiologie 95, 69-80.

Gabriel, W. \& Lynch, M. (1992) The selective advantage of reaction norms for environmental tolerance. Journal of Evolutionary Biology 5, 41-59.

Gabriel, W. \& Taylor, B.E. (1991) Optimal resource allocation in cladocerans. Internationale Vereinigung für Theoretische und Angewandte Limnologie Verhandlungen 24, 2784-2787.

Gurney, W.S.C., McCauley, E., Nisbet, R.M. \& Murdoch, W.W. (1990) The physiological ecology of Daphnia: a dynamic model of growth and reproduction. Ecology 71, 716-732.

Hallam, T.G., Lassiter, R.R., Li, J. \& Suarez, L.A. (1990) Modelling individuals employing an integrated energy response: application to Daphnia. Ecology 71, 938-954.

Hebert, P.D.N. (1978) The population biology of Daphnia (Crustacea, Daphnidae). Biological Reviews of the Cambridge Philosophical Society 53, 387-426.

Iwasa, Y. \& Roughgarden, J. (1984) Shoot/root balance of plants: optimal growth of a system with many vegetative organs. Theoretical Population Biology 25, 78-105.

Jacobs, J. (1987) Cyclomorphosis in Daphnia. Memorie dell'Istituto Italiano di Idrobiologia Dott. Marco de Marchi 45, 325-352.

Keyfitz, N. (1977) Introduction to the Mathematics of the Population (with Revisions). Addison-Wesley Publishing Company, Reading, Massachusetts.

King, D. \& Roughgarden, J. (1982) Graded allocation between vegetative and reproductive growth for annual plants in growing seasons of random length. Theoretical Population Biology 22, 1-16.

Kleiven, O.T., Larsson, P. \& Hobæk, A. (1992) Sexual reproduction in Daphnia magna requires three stimuli. Oikos 65, 197-206.

Kozlowski, J. \& Ziólko, M. (1988) Gradual transition from vegetative to reproductive growth is optimal when the maximum rate of reproductive growth is limited. Theoretical Population Biology 34, 118-129.

León, J.A. (1976) Life histories as adaptive strategies. Journal of Theoretical Biology 60, 301-335.

Lynch, M. (1980) The evolution of cladoceran life histories. Quarterly Review of Biology 55, 23-42.

Perrin, N. (1992) Optimal resource allocation and the marginal value of organs. American Naturalist 139, 1344-1369.

Perrin, N., Ruedi, M. \& Saiah, H. (1987) Why is the cladoceran Simocephalus vetulus (Müller) not a 'bangbang strategist'? A critique of the optimal-body-size model. Functional Ecology 1, 223-228.

Pugliese, A. (1987) Optimal resource allocation and optimal size in perennial herbs. Journal of Theoretical Biology 126, 33-49.

Pugliese, A. (1988a) Optimal resource allocation in perennial plants: a continuous-time model. Theoretical Population Biology 34, 215-247.

Pugliese, A. (1988b) Optimal life history models: effects of nonlinearities in the response of reproductive success to investment. Biomathematics and Related Computational Problems (ed. L. M. Ricciardi), pp. 223-235. Kluwer Academic Publishers, Dordrecht. 
Optimal growth of

Daphnia
Richman, S. (1958) The transformation of energy by Daphnia pulex. Ecological Monographs 28, 273-291.

Schaffer, W.M. (1983) The application of optimal control theory to the general life history problem. American Naturalist 121, 418-431.

Sibly, R., Calow, P. \& Nichols, N. (1985) Are patterns of growth adaptive? Journal of Theoretical Biology 112, 553-574.

Spitze, K. (1992) Predator-mediated plasticity of prey life history and morphology: Chaoborus americanus predation on Daphnia pulex. American Naturalist 139, 229-247.

Stearns, S.C. (1976) Life-history tactics: a review of the ideas. Quarterly Review of Biology 51, 3-47.

Stibor, H. (1992) Predator induced life-history shifts in a freshwater cladoceran. Oecologia 92, 162-165.

Taylor, B.E. (1985) Effects of food limitation on growth and reproduction of Daphnia. Archiv für Hydrobiologie Beihefte 21, 285-296.
Taylor, B.E. \& Gabriel, W. (1992) To grow or not to grow: optimal resource allocation for Daphnia. American Naturalist 139, 248-266.

Taylor, B.E. \& Mahoney, D.L. (1990) Zooplankton in Rainbow Bay, a Carolina bay pond: population dynamics in a temporary habitat. Freshwater Biology 24, 597-612.

Threlkeld, S.T. (1987) Daphnia life history strategies and resource allocation patterns. Memorie dell'Istituto Italiano di Idrobiologia Dott. Marco de Marchi 45, 353-366.

Vincent, T.L. \& Pulliam, H.R. (1980) Evolution of life history strategies for an asexual annual plant model. Theoretical Population Biology 17, 215-231.

Received 27 April 1992; revised 20 January 1993; accepted 5 April 1993 